\title{
Web Objects Ambient: an integrated platform supporting new kinds of Personal Web experiences
}

\author{
Sergio Firmenich ${ }^{1}$, Gabriela Bosetti ${ }^{1}$, Gustavo Rossi ${ }^{1}$, Marco Winckler ${ }^{2}$ \\ ${ }^{1}$ LIFIA, Facultad de Informática, Universidad Nacional de La Plata \\ \{sergio.firmenich, gabriela.bosetti, \\ gustavo\} @lifia.info.unlp.edu.ar \\ 2 ICS-IRIT, University of Toulouse 3, France \\ winckler@irit.fr
}

\begin{abstract}
The Personal Web arose to empower end users with the ability to drive and integrate the Web by themselves, according to their own interests. This is usually achieved through Web Augmentation, Mashups, Personal Information Managers (PIM), etc. But despite the diverse approaches, there are still scenarios that require to be solved in combination of more than one of these approaches; this implies the end user knowing more than one tool and being able to coordinate them. This paper presents WOA, a platform conceived under the foundations of the Personal Web, for supporting the collection and materialization of information objects from existing Web content, and their enhancement through the addition of specialized behaviour. This makes it possible to conceive multiple Web information objects coexisting in a same space of information and offering the end user with different modes of interaction, therefore, with multiple kinds of personal Web experiences.
\end{abstract}

Keywords: Personal Web, Client-Side Adaptation, Web APIs.

\section{Introduction}

The web content is daily growing in number and diversity, and it is increasingly likely that much of the information we need for our daily activities is already available somewhere on the Web. There is a myriad of tasks the user can perform by consuming information from different sources. For example, consider a radio journalist consuming news from a concrete Web portal and related posts from social networks to stay informed about public user opinions, to eventually analyse them and produce some content for his podcast. In a similar way we can think of planning a trip, comparing prices and reviews among products, organizing events according to weather conditions, seeking answers among different user communities, etc.

Several approaches have addressed this need to consume and combine information from different sources for performing daily life tasks. Literature about Mashups [2] presents a vast number of examples supporting this claim. Another kind of application

adfa, p. 1, 2011 .

(C) Springer-Verlag Berlin Heidelberg 2011 
supporting this is a Personal Information Management (PIM) [3], which allow the user to collect objects for further making them available for performing specific operations. Reactive Web [5] could also be considered, as it allows propagating changes in -even remote- information objects, so specific behaviour could be associated to handle the up-to-date retrieved data. It is also common to read or to be involved in situations in which we count with the necessary data to automatically solve a problem -not necessarily provided by different sources-, but not with an associated mechanism to operate them. This is where come into play Web Augmentation [1], a technique for enhancing existing Web pages according to the user's requirements; this includes adding -or adapting-specialized behaviour, styles, content, etc. If this technique is implemented as some kind of privileged script, such as Greasemonkey or browser extensions, it is also possible to include some external Web pages' content as part of the augmentation. Finally, it is also worth mentioning specific domain applications designed to use existing data objects, such as the ones for managing scientific literature, like Mendeley ${ }^{1}$.

Each of the mentioned applications and techniques seem to empower users with the capability of interacting with existing content, but working isolated; therefore, making it difficult to have a complete Personal Web experience. The Personal Web, as explained in [4], is intended to "empower ME, as a common internet user of generally limited technical skills, the autonomy and ease of control in assembling and aggregating integrate-able web elements across the web for a particular sphere of context of my concern". This promises to completely change the user experience in the WWW, because now he is the centre of gravity of such space of information.

In this light, we propose an approach for achieving Personal Web through diverse but combinable implementation strategies that eventually allow diverse modes of user interactions with the created objects under a common ambient. This way it is possible to consider a full interactive Personal Web experience, by reusing existing Web content and improving them with specialized behaviour artefacts, already available in the ambient. This approach consider two user roles: an end user and a developer. The first one is empowered with the capability of 1) collecting and materializing existing information objects in the Web and 2) associating them some predefined specialized behaviour, for further 3 ) interacting with the created objects in different ways and contexts. The second one is in charge of providing end users with new specialized behaviour artefacts, in the means of 1) decorators and 2) concrete client-side domain-specific applications. Web Objects Ambient (WOA) is the implemented platform supporting this approach, and we present it as the contribution of this demo paper.

\section{WOA in a nutshell}

WOA is composed by specialized mechanisms for collecting Web content and materializing it into concrete information objects and a common objects environment where these lived once materialized, as shown at the left side of Figure 1. Then, as shown at right, it is possible to enhance such instances with specialized decorators -

1 Mendeley: https://www.mendeley.com/ 
whose classes can be extended by developers-. That enables the user interaction with the objects through the Objects Viewer or Web Augmentation decorators, or through specialized applications -implemented by developers by now-.

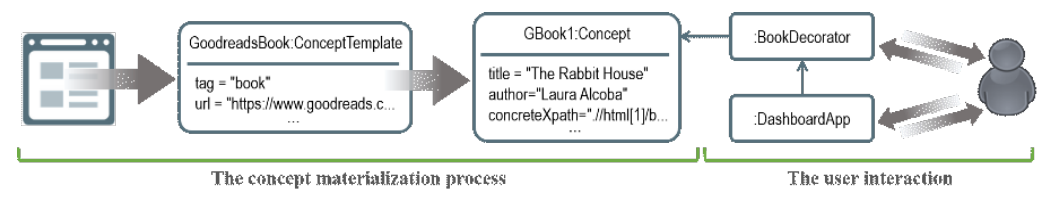

Fig. 1. WOA supporting end user interactions with information objects

Matching between decorators and objects is defined by using both semantic and enduser-defined tags.

\subsection{The concept materialization process}

Collecting objects into the WOA allow end users to interact with them in different ways and contexts, enabling new ways of Personal Web experiences, but also and turning part of the Web -the part that interests the user- into a holistic space of information. We call concept materialization to the process consisting in (1) identifying information objects of interest for the user, in the context of any Web page, (2) abstracting them by giving them a semantic tag, (2) structuring the abstraction, which involves setting its internal state, mapping it with the proper DOM element and obtaining a template (an Object Model Specification) and finally (3) extracting the available concept instance(s) by interpreting the specification of such template. You can see these steps in Figure 2.

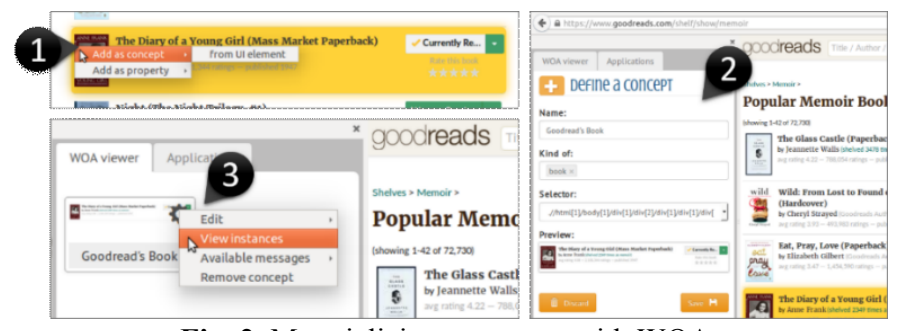

Fig. 2. Materializing a concept with WOA

\subsection{Interacting with the extracted objects}

Once a template has been defined, you can appreciate in Figure 3 that the user can interact with the extracted concept templates and instances through the WOA Viewer (1) or in-situ modality, by using Web augmentation decorators (2). If the retrievable instances are not presented in a single DOM (there is a navigation bar or a search engine), the user has the possibility to configure a WOA search engine (3), so he can browse and interact with all the instances provided by the site. Finally, he can also interact with the instances by importing a WOA application (4); a regular Web application that uses the WOA API for accessing the collected concepts and decorators. 
It is worth mentioning that developers have the possibility of extending decorators whether or not in-situ- and develop their own WOA applications, which can be easily imported to be used in the ambient.

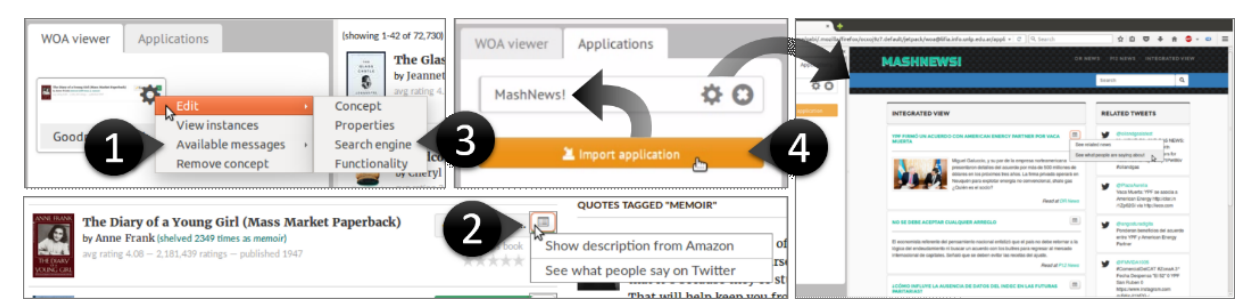

Fig. 3. Forms of interaction with ambient concepts

\section{Conclusion}

In this paper we have introduced WOA, a platform supporting a novel approach for enabling end users to create a diverse range of Web Personal experiences, by abstracting and structuring existing Web content. We fully implemented the common ambient and a set of collectors and decorators to demonstrate the feasibility of the approach. Now, we are extending the collectors in order to support new extraction mechanisms, and also the decorators to provide the end users with a broader spectrum of specific functionality. We are also working on the implementation of an end user tool for empowering them with the capability of producing their own Dashboard applications. Finally, we are designing the first experiment of the approach, focused on end users. For further details about WOA, please visit our project Web site ${ }^{2}$.

\section{$4 \quad$ References}

1. Díaz, O.; Arellano, C. The Augmented Web: Rationales, Opportunities, and Challenges on Browser-Side Transcoding. ACM Transactions on the Web, 2015, vol. 9, no 2, p. 8.

2. Ennals, R., Garofalakis, M. Mashmaker: Mashups for the masses (demo paper). In Proceedings of the 2007 ACM SIGMOD International Conference on Management of Data (SIGMOD'2007).

3. Karger, D. R., Bakshi, K., Huynh, D., Quan, D., \& Sinha, V. (2005, January). Haystack: A customizable general-purpose information management tool for end users of semistructured data. In Proc. of the CIDR Conf.

4. Ng, J. (2010, November). The Personal Web: smart internet for me. In Proceedings of the 2010 Conference of the Center for Advanced Studies on Collaborative Research (pp. 330344). IBM Corp.

5. Van Kleek, M., Moore, B., Karger, D. R., \& André, P. (2010, April). Atomate it! End-user context-sensitive automation using heterogeneous information sources on the web. In Proceedings of the 19th International Conference on World Wide Web (pp. 951-960). ACM.

2 WOA documentation Web site: https://sites.google.com/site/webobjectambient/ 